\title{
Charge trapping in amorphous dielectrics for secure charge storage
}

Seung Jae Baik ${ }^{1, *}$ and Hyunjung Shin, ${ }^{2, *}$

${ }^{1}$ Department of Electrical, Electronic, and Control Engineering, Hankyong National

University, 327 Jungang-ro, Anseong-si, Gyeonggi-do, 17579 Korea

2Department of Energy Science, Sungkyunkwan University, 2066 Seobu-ro, Suwon-

si, Gyeonggi-do, 16419 Korea

KEYWORDS : NAND flash, Charge trap flash, Amorphous, Dielectric constant,

Composition grading

*Author to whom correspondence should be addressed: E-mail: sjbaik@hknu.ac.kr,

hshin@skku.edu 
S-2 
Figure S1 Glancing incidence X-day diffraction (GIXRD) spectra with incident angle 3.0 $0^{\circ}$ GIXRD spectra of as-deposited $\mathrm{Hf}_{x} \mathrm{Al}_{1-\mathrm{x}} \mathrm{O}_{\mathrm{y}}$ thin films (a) and post-deposition annealed $\mathrm{Hf}_{x} \mathrm{Al}_{1-\mathrm{x}} \mathrm{O}_{y}$ thin films (b). After annealing, $\mathrm{HfO}_{2}$ and $\mathrm{Hf}_{0.7} \mathrm{Al}_{0.3} \mathrm{O}_{\mathrm{y}}$ show crystalline peaks and annealed films with $x$ smaller than 0.7 does not show any signature of crystallization.

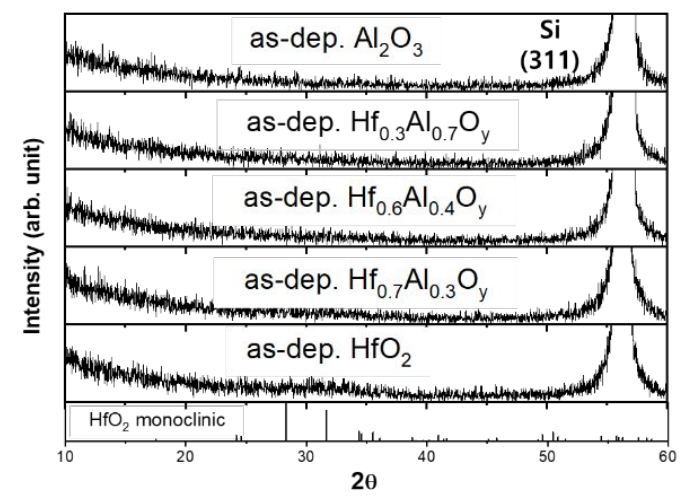

(a)

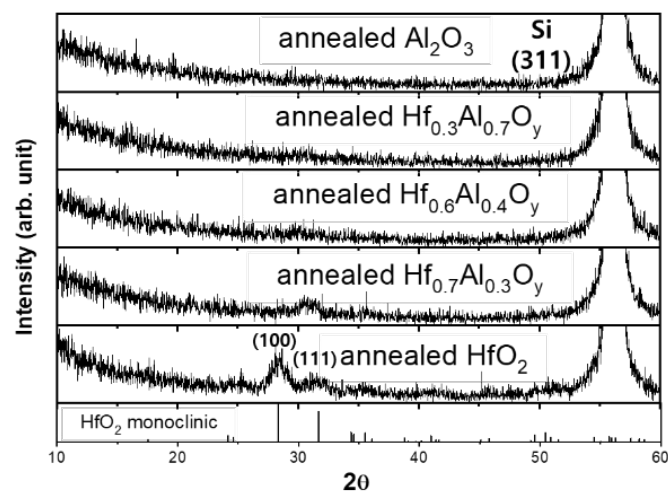

(b)

Figure S2 Equivalent oxide thickness (EOT) measured from TiN/Hf $\mathrm{HAl}_{1-\mathrm{x}} \mathrm{O}_{\mathrm{y}} 6 \mathrm{~nm} / \mathrm{SiO}_{2}$ $4 \mathrm{~nm} / \mathrm{Si}$ capacitors. (a) EOT vs. $x$ for the capacitors with as-deposited (solid rectangle) and post-deposition annealed (open rectangle) uniform $\mathrm{Hf}_{\mathrm{x}} \mathrm{Al}_{1-\mathrm{x}} \mathrm{O}_{\mathrm{y}}$. (b) EOT vs. $\mathrm{x}$ for the capacitors with as-deposited (solid rectangle) and post-deposition annealed (open rectangle) band engineered $\mathrm{Hf}_{x} \mathrm{Al}_{1-x} \mathrm{O}_{y}$. The dielectric constant of as-deposited $\mathrm{Hf}_{x} \mathrm{Al}_{1}$ ${ }_{x} \mathrm{O}_{y}$ thin films ranges from 10.2 to 15.6 as $x$ increases. Post-deposition annealing causes crystallization induced dielectric constant increment, and possibly causes oxidation of substrate Si. The effect of crystallization is EOT decrease and the effect of oxidation is EOT increase, the combination of both effects produces EOT trend with $x$ variation for annealed samples. 

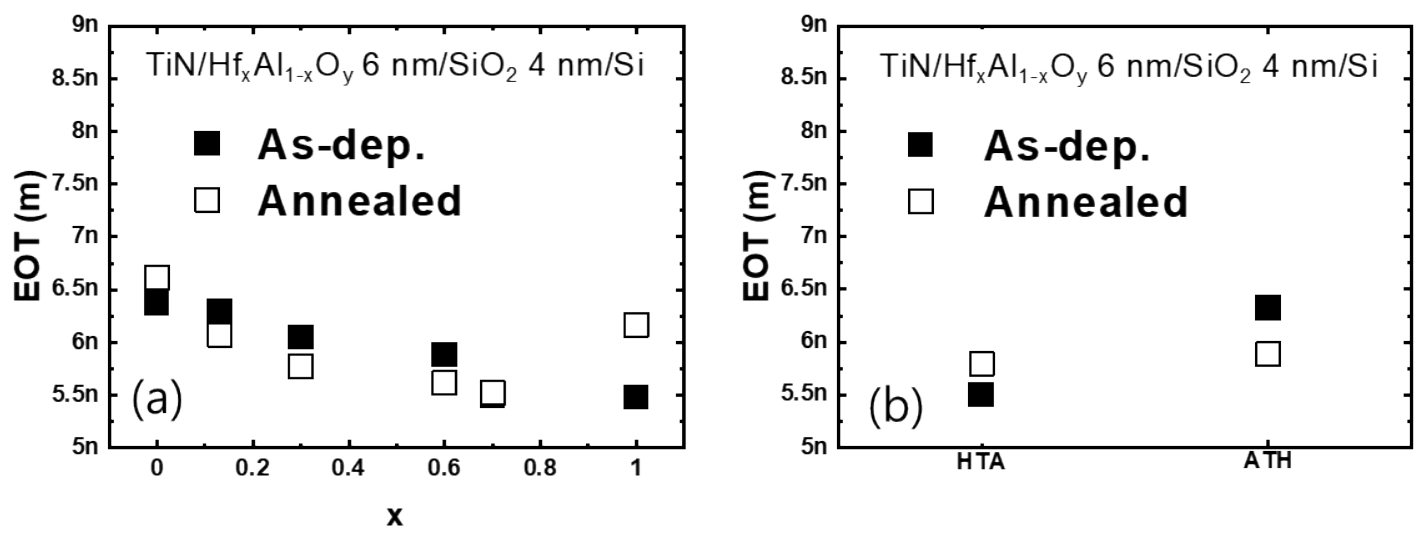

Figure S3 Bias dependent charge retention characteristics from capacitor devices with post-deposition annealed ATH (a) and HTA (b).
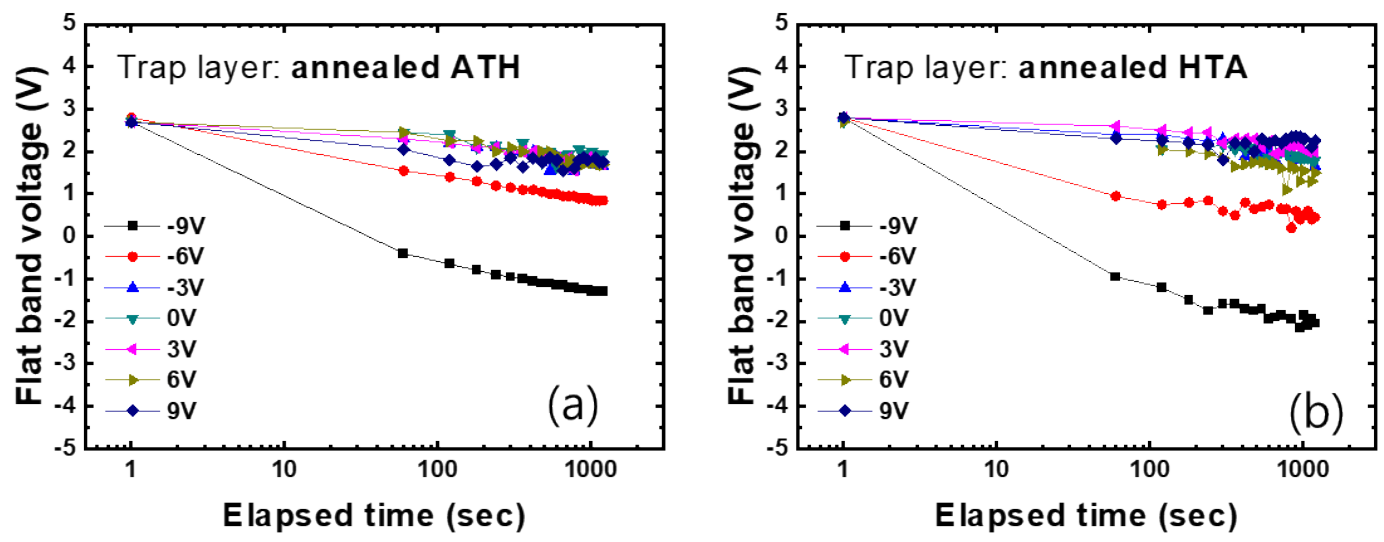